\title{
Bronchopulmonary dysplasia: rationale for a pathophysiological rather than treatment based approach to diagnosis.
}

\author{
Benjamin Stoecklinn ${ }^{a, b, c}$, Shannon J. Simpson ${ }^{d}$, Jane J. Pillow ${ }^{a, b^{*}}$ \\ ${ }^{a}$ School of Human Sciences, University of Western Australia, Perth, Australia \\ ${ }^{b}$ Centre for Neonatal Research and Education, University of Western Australia, Perth, Australia; \\ c Neonatal Clinical Care Unit, King Edward Memorial Hospital, Perth, Australia \\ d Telethon Kids Institute, Perth, Australia \\ * Corresponding author. School of Human Sciences, University of Western Australia, 35 Stirling \\ Highway, Perth WA 6009, Australia. E-mail address: jane.pillow@uwa.edu.au
}

\section{Funding sources}

Dr Stoecklin was supported by the Swiss National Science Foundation (P2BSP3_158837) and a Research Training Program scholarship, The University of Western Australia. Prof Jane Pillow is supported by a National Health Medical Research Council Senior Research Fellowship (NHMRC 1077691).

Financial disclosure

None of the authors have financial relationships to this article relevant to disclose.

Directions for future research

Develop diagnostic criteria for BPD based on quantitative function rather than unstandardized treatment, to reflect more accurately the pathophysiological basis and severity of BPD and to provide meaningful insights that guide ongoing cardiorespiratory management.

\section{Educational aims}

- Definition of bronchopulmonary dysplasia across the ages

- Pitfalls of the current definition of bronchopulmonary dysplasia

- Shift as a marker of impaired pulmonary gas exchange capacity

- Shift of the $\mathrm{SpO}_{2} / \mathrm{P}_{1} \mathrm{O}_{2}$ curve as a dynamic physiological test for severity of bronchopulmonary dysplasia

\section{Summary}

Bronchopulmonary Dysplasia (BPD), also known as Chronic Lung Disease (CLD), is a chronic respiratory condition of prematurity with potential life-long consequences for respiratory well-being. BPDwas first described by Northway in 1967, when the mean gestation of preterm infants with BPD was 34 weeks' postmenstrual age (PMA). Survival of preterm infants at lower gestational ages has increased steadily since 1967 associated with marked improvements in respiratory management of respiratory distress syndrome. Currently, BPD develops in approximately $45 \%$ of all infants born extremely preterm [1]. These smaller and more immature babies are born during the late canalicular or early saccular period of lung development. Not surprisingly, the pathophysiology of BPD also evolved since classical BPD was described. As the nature and our understanding of BPD evolved, so too the definitions and classification of BPD changed over time. These differing and ever-changing definitions hamper clinical benchmarking as they are interpreted and applied inconsistently, and 
define BPD and its severity by non-standardised treatments rather than independent evaluations of structure or function. A standardised, unambiguous definition and classification of BPD is essential for evaluation and improvement in clinical practice, both within an individual unit, as well as across and between neonatal networks. The determination and implementation of diagnostic criteria and severity classification that is standardised, globally applicable, and that has prognostic utility for clinical outcomes and guidance of ongoing respiratory management remains of utmost importance. This review describes the evolution of BPD definitions, evaluates the benefits and limitations of each approach, and discusses alternative approaches that may improve the functional assessment of BPD severity.

Keywords

Infant, premature

Bronchopulmonary Dysplasia

Pulmonary Gas Exchange

Infant, Newborn, Diseases

Oximetry

Physiological definition

Abbreviations: BPD, bronchopulmonary dysplasia; PMA, postmenstrual age; NICHD, National Institute of Child Health and Human Development; PNA, postnatal age; $\mathrm{F}_{1} \mathrm{O}_{2}$, fractional inspired oxygen; PPV, positive pressure ventilation; $\mathrm{CPAP}$, continuous positive airway pressure; $\mathrm{P}_{1} \mathrm{O}_{2}$, inspired oxygen pressure

\section{Definitions of BPD across the ages}

\section{The original definition of BPD}

The original definition of BPD was published by Northway in 1967. Affected infants were born during the late saccular or the alveolar stage of lung development and exposed to high oxygen concentrations and high peak airway pressure during mechanical ventilation $[2,3]$.

Northway described 4 stages of BPD based on the clinical, radiographic and the histopathological findings (Table 1) [4]. Preterm infants with BPD initially presented with acute respiratory distress syndrome, which evolved to a chronic disease after day 28 of life. The chest radiograph findings correlated with changes in histopathology, which showed an initial acute inflammatory stage

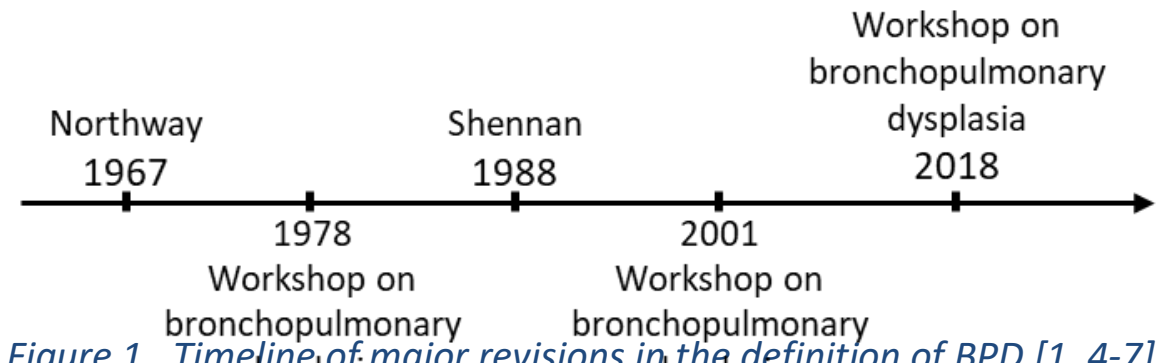

Figure 1. Timedjsplafiamajor revisions inthspadefinition of BPD [1, 4-7]. 
Table 1. Stages of bronchopulmonary dysplasia described by Northway et al. in 1967.

\begin{tabular}{lll} 
BPD Stages & Chest radiograph & Histopathology (most significant findings) \\
\hline Stage I & Generalised granular & Hyaline membranes, atelectasis, lymphatic \\
Acute respiratory & pattern, air bronchogram. & dilatation. \\
distress syndrome & & Decrease in ciliated cells with metaplasia \\
$(2-3$ days $)$ & & and necrosis of bronchiolar mucosa.
\end{tabular}

$\begin{array}{lll}\text { Stage II } & \text { White lungs, no visible } & \text { Persisting hyaline membranes. } \\ \text { Period of } & \text { cardiac borders. } & \text { Necrosis of alveolar epithelium and } \\ \text { regeneration } & \text { bronchioles together with repair of } \\ \text { (4-10 days) } & \text { epithelium. } \\ & \text { Focal thickening of capillary basement } \\ & \text { membranes. }\end{array}$

$\begin{array}{lll}\text { Stage III } & \text { Small rounded areas of } & \text { Decrease in hyaline membranes. } \\ \text { Transition to chronic } & \text { radiolucency across both } & \text { Persisting injury to alveolar epithelia. } \\ \text { disease } & \text { lungs coexistent with areas } & \text { Mucous secretion, moderate exudation of } \\ (10-20 \text { days }) & \text { of irregular density. } & \text { alveolar macrophages and histiocytes. } \\ & \text { No signs of cardiomegaly } & \text { Focal thickening in the basement } \\ & \text { and no air bronchogram. } & \text { membrane. }\end{array}$

$\begin{array}{lll}\begin{array}{l}\text { Stage IV } \\ \text { Chronic disease }\end{array} & \begin{array}{l}\text { Enlargement of the rounded } \\ \text { areas described in stage III. }\end{array} & \begin{array}{l}\text { Increased number of macrophages, } \\ \text { histiocytes, foam cells. }\end{array} \\ & \begin{array}{l}\text { Irregular density areas } \\ \text { thinner than in stage III. }\end{array} & \begin{array}{l}\text { Permucosal fibrosis, metaplasia with } \\ \text { detaching mucosal cells. }\end{array} \\ & \begin{array}{l}\text { Cardiomegaly } \\ \text { alved separation of capillaries from } \\ \end{array} & \text { Early signs of pulmonary hypertension } \\ & \text { (e.g. medial hypertrophy). }\end{array}$

followed by chronic inflammatory processes that resulted in fibrosis. A timeline highlighting the most important revisions to the definition is shown in Figure 1. 


\section{BPD definition during the pre-surfactant era}

Northway's definition of BPD was revised in the late 1970s [5], motivated by overlap and inconsistencies in the radiologic findings between acute respiratory distress syndrome and advanced stages of BPD. Moreover, the four consecutive radiologic stages of BPD described by Northway were observed rarely. The new definition provided a more functional definition of BPD based on longterm abnormal radiologic findings at 30 days of life and oxygen dependency at 28 days of life $[6,7]$. Ten years later, chest radiograph findings were abolished from the BPD definition, as the pulmonary radiologic changes did not always correlate with disease severity [8]. Shennan et al. showed that oxygen dependency at 28 days was a poor predictive factor for negative pulmonary outcomes in infants born before 30 weeks' gestation and instead suggested assessment of oxygen dependency at 36 weeks' PMA. The revised definition was published in 1988 and was restricted to infants with a birthweight $<1500 \mathrm{~g}$, reflecting the transition of BPD to a more immature preterm infant group [9]. At this time the terminology of Chronic Lung Disease (CLD) was introduced as an alternative

\section{The post-surfactant era}

The introduction of antenatal maternal glucocorticoids and postnatal exogenous surfactant therapy in the early 1970s and the early 1990s respectively, enabled survival of infants born extremely preterm. Extremely preterm infants are born with lungs in the late canalicular or very early saccular stage of development, which changed the underlying pathophysiology of BPD [10]. The immature lungs affected by BPD showed fewer alveoli with less fibrosis and an overall more diffuse disease of the lung parenchyma. Jobe coined the term 'new BPD' in 1999, [11], to reflect the histopathological changes in BPD seen in the surfactant era $[8,12,13]$.

In contrast to Northway's original description of BPD, the new BPD is a consequence of arrested lung development secondary to a chronic inflammatory process after respiratory distress syndrome [1316]. In 2001, the National Institute of Child Health and Human Development (NICHD) published a consensus on the definition of BPD reflecting the changes the pathophysiology and management of BPD over time (Table 2) [8]. The NICHD criteria included a severity based scale for the first time, dependent on the oxygen requirement of the preterm infant at 36 w PMA (or at discharge). The 2001 definition reflected the improvement in respiratory care responsible for the fact, that BPD now almost only affected infants born $<32$ weeks' gestational age and / or a birth weight $<1200 \mathrm{~g}$ [17]. 
Table 2. NICHD criteria for severity of Bronchopulmonary Dysplasia

Pre-requisite for BPD (any severity): $\quad \mathrm{F}_{1} \mathrm{O}_{2}>0.21$ for at least $28 \mathrm{~d}$

BPD Severity

\begin{tabular}{llll}
\hline GA (w PMA) and assessment timing & Mild & Moderate & Severe \\
\hline$<32$ Sooner of $36 \mathrm{w}$ PMA or & $\mathrm{F}_{1} \mathrm{O}_{2}=0.21$ & $0.21>\mathrm{F}_{1} \mathrm{O}_{2}<0.30$ & $\mathrm{~F}_{1} \mathrm{O}_{2}=0.30$ and/or positive \\
discharge home & & pressure (PPV or nasal \\
& & & CPAP) \\
$\geq 32 \quad \begin{array}{l}\text { Sooner of }>28 \mathrm{~d} \text { but }<56 \mathrm{~d} \\
\text { PNA, or discharge home }\end{array}$ & $\mathrm{F}_{1} \mathrm{O}_{2}=0.21$ & $0.21>\mathrm{F}_{1} \mathrm{O}_{2}<0.30$ & $\mathrm{~F}_{1} \mathrm{O}_{2}=0.30$ and/or positive \\
& & & pressure (PPV or nasal \\
& & CPAP)
\end{tabular}

PMA, postmenstrual age; PNA, postnatal age; $\mathrm{F}_{1} \mathrm{O}_{2}$, fractional inspired oxygen; PPV, positive pressure ventilation; CPAP, continuous positive airway pressure. Adapted from ([8].

Walsh et al. proposed a physiological classification derived from an oxygen reduction test in 2003 [18]. In contrast to the semi-quantitative severity-based NICHD definition published in 2001, the outcome of the Walsh test is binary. Infants failing the oxygen reduction test are classified as having $\mathrm{BPD}$, whereas infants who pass the test are considered to not have BPD. The $\mathrm{SpO}_{2}$ cut-off value for passing the test was set to $\geq 90 \%$. However, normal $\mathrm{SpO}_{2}$ levels in healthy infants are $>96 \%$ [19]. Therefore, preterm infants with mild impairment in oxygenation are misclassified as healthy, when using the Walsh criteria. In practice, the Walsh test is sometimes used to determine if BPD is present, with the 2001 NICHD classification added to grade disease severity.

The debate over the definition and classification has continued, with a recent cluster of opinion and review articles discussing the relative merits of different approaches to diagnosis of BPD [20-23]. In a summary of the 2016 NICHD Workshop on BPD, the workshop panel proposed a further revision of the BPD definition in April 2018 [24]. The proposed new definition no longer includes the assessment of oxygen use for 28 days prior to 36 weeks PMA and emphasises the new modes of non-invasive respiratory support including nasal cannula. In contrast to the 1988 and 2001 definitions, abnormal chest radiograph findings are again included in the definition. The subjective 
terminology of mild, moderate and severe BPD is replaced by grades I-III. Infants who die due to a respiratory cause prior to 36 weeks PMA are reflected in the categorisation as grade III(A). The grading depends on the amount of oxygen the infants receives for at least three consecutive days around 36 weeks PMA, type of assisted support and also on the flows of supplemental nasal cannula gas when used (Table 3$)$.

Table 3. April 2018 revision of the 2001 NICHD BPD criteria

\begin{tabular}{|c|c|c|c|c|}
\hline \multirow[t]{2}{*}{ Grade } & \multicolumn{4}{|l|}{ BPD Severity Grade } \\
\hline & I & II & III & IIIA \\
\hline Invasive PPV* & - & 0.21 & $>0.21$ & $>0.21$ \\
\hline nCPAP, NIPPV, or & 0.21 & $>0.21$ but $<0.30$ & $\geq 0.30$ & Early death \\
\hline nasal cannula $\geq 3$ & & & & (between $14 \mathrm{~d}$ PNA \\
\hline $\mathrm{L} / \mathrm{min}$. & & & & and 36 w PMA) \\
\hline & & & & owing to persistent \\
\hline Nasal cannula: & $>0.21$ but $<0.30$ & $\geq 0.30$ & & parenchymal lung \\
\hline $1 \mathrm{~L} / \mathrm{min} \leq$ flow $<$ & & & & disease and \\
\hline $3 \mathrm{~L} / \mathrm{min}$ & & & & respiratory failure \\
\hline & & & & not attributable to \\
\hline Nasal cannula & $>0.21$ but $<0.70$ & $\geq 0.30$ & & other neonatal \\
\hline flow $\geq 3 \mathrm{~L} / \mathrm{min}$ & & & & morbidities \\
\hline $\mathrm{Hood} \mathrm{O}_{2}$ & $>0.21$ but $<0.30$ & $\geq 0.30$ & & \\
\hline
\end{tabular}

Data in cells are proposed $\mathrm{FiO}_{2}$ limits. * excludes infants ventilated for primary airway disease or central respiratory control conditions. PPV, positive pressure ventilation; nCPAP, nasal continuous positive airway pressure; NIPPV, non-invasive positive pressure ventilation; PNA, postnatal age; PMA, postmenstrual age. Adapted from Higgins et al. [24].

\section{Pitfalls of the current BPD definitions}

The 2001 and current NICHD BPD classification have multiple pitfalls, as outlined and briefly discussed below. 


\section{1) Use of treatment as basis for definition and classification:}

The definition of BPD by its treatment rather than the underlying pathophysiology and its consequences for later outcomes is perplexing and fundamentally flawed [25]. The non-standardised target range for $\mathrm{SpO}_{2}$ used to determine prescription of supplemental oxygen leads to global differences in oxygen prescribing policies [23, 26-28]. Similarly, departmental guidelines and physician practices for choice of mechanical respiratory support, and duration of such treatments vary widely. Not surprisingly, the incidence of BPD defined by such treatments also varies widely [29]. Importantly, neither level of oxygen therapy or type/duration of respiratory support at $36 \mathrm{w}$ PMA properly inform clinicians regarding the specific nature of the underlying problem and hence do not assist development of appropriate clinical management.

\section{2) Semi-quantitative assessment and arbitrary thresholds:}

The severity of BPD is a semi-quantitative assessment based on arbitrary thresholds. Infants are categorized into three different stages based on the level of respiratory support and physicianprescribed oxygen dependency at 36 weeks' PMA. Semi-quantitative categories are useful for broad classification but limit sensitivity for detection of clinically relevant differences between intervention groups (in a randomised controlled trial) or for determination of improvement in condition for any given infant over time. Ideally, thresholds for classifications levels should be modelled from prognostic outcomes for survival and long-term morbidities in a way that would guide ongoing management. Examples of the arbitrary nature of thresholds in the most recent NICHD skeletal definition of BPD are the different inspired oxygen thresholds for infants on nasal cannulae compared to oxygen hood, and inconsistencies in whether threshold values belong to the lower or higher category.

\section{3) Failure to consider altitude in assessment of BPD:}

The availability of oxygen in inspired gas mixtures is dependent on altitude (Table 3) [30, 31]. The role of altitude in the assessment of BPD using current definitions is revealed by examination of the alveolar gas equation. The simplified version of the alveolar gas equation equals $\mathrm{PaO}_{2}=\mathrm{P}_{I} \mathrm{O}_{2}-$ $\frac{\mathrm{PaCO}_{2}}{R}$, where $\mathrm{PaO}_{2}$ and $\mathrm{PaCO}_{2}$ are the partial pressure of $\mathrm{O}_{2}$ and $\mathrm{CO}_{2}$ in the arterial blood, respectively. $P_{1} \mathrm{O}_{2}$ is the partial inspired oxygen pressure and $\mathrm{R}$ is the respiratory exchange ratio [32]. $P_{1} \mathrm{O}_{2}$ decreases with an increase in altitude: a lower $P_{1} \mathrm{O}_{2}$ results in a decrease in $\mathrm{PaO}_{2}$ and 
subsequently in $\mathrm{SpO}_{2}$ (Table 3). Therefore, the incidence of BPD increases with increasing altitude above sea level, unless adequate adjustment is made for the $P_{1} O_{2}[33,34]$.

Table 4. Oxygen availability dependent on altitude above sea level

\begin{tabular}{llllll}
\hline Altitude $(\mathrm{m})$ & $\begin{array}{l}\text { Barometric } \\
\text { pressure }(\mathrm{kPa})\end{array}$ & $\begin{array}{l}\text { Barometric } \\
\text { pressure }(\mathrm{mmHg})\end{array}$ & $\mathrm{F}_{1} \mathrm{O}_{2}$ & $\mathrm{P}_{1} \mathrm{O}_{2}(\mathrm{kPa})$ & $\begin{array}{l}\text { \% Oxygen } \\
\text { availability }\end{array}$ \\
\hline 0 (sea level) & 101 & 760 & 0.21 & 21 & 100 \\
200 & 99 & 743 & 0.21 & 20.8 & 98 \\
500 & 96 & 718 & 0.21 & 20.2 & 94 \\
1000 & 90 & 679 & 0.21 & 18.9 & 89 \\
2000 & 81 & 604 & 0.21 & 17.0 & 80 \\
\hline
\end{tabular}

$P_{1} \mathrm{O}_{2}=\mathrm{F}_{1} \mathrm{O}_{2}$ (barometric pressure $-47 \mathrm{mmHg}$ ), where $\mathrm{P}_{1} \mathrm{O}_{2}$ is partial pressure of inspired oxygen and $\mathrm{F}_{1} \mathrm{O}_{2}$ is fractional inspired oxygen.

\section{4) Interpretation of Chest Radiographs}

Abnormal chest radiograph findings were the most important factor of the initial definition of BPD described by Northway in 1967. Serial chest radiographs showed the progress of the disease [2]. However, the radiologic changes did not follow a strict pattern with an increase of survival of younger preterm infants, and were therefore abolished in the 1988 and 2001 definitions [8, 9, 35]. Surprisingly, the 2018 revision reintroduces a requirement for radiographic confirmation of parenchymal lung disease in the definition [21]. An explanation for this reversion is not provided, but radiographic changes may have been included to exclude diagnosis of BPD from increased ventilation and oxygen requirements due solely to extrapulmonary factors such as cardiac, airway, respiratory muscles pathology or respiratory control. 
Pitfalls 1 - 4 above lead to differences in how BPD is assessed globally resulting directly from nonstandardised $\mathrm{SpO}_{2}$ target range, non-inclusion of altitude as an important determinant of $P_{1} \mathrm{O}_{2}$ and inconsistencies in the interpretation of chest x-rays. Consequently, the current definitions do not provide a useful tool for clinical benchmarking between neonatal units from different geographic regions.

\section{6) Time-points for diagnosis of BPD and assessment of its severity:}

Debate continues regarding appropriate time-points for diagnosis of BPD, and for the assessment of its severity. The requirement for treatment with supplemental oxygen for at least $28 d$ in the 2001 $\mathrm{NICHD}$ definition was interpreted variably as 28 total days of oxygen support or oxygen support at day 28 , including either any supplemental oxygen on any of those days or at least 12 hours supplemental oxygen on any day defined as a day of oxygen therapy. Furthermore, this stipulation precluded diagnostic classification of an infant prior to $28 \mathrm{~d}$, and hence had limited utility as a criterion for institution of higher-order therapies. The recent revised NICHD definition of BPD removed the requirement for 28 days of oxygen therapy prior to 36 weeks PMA, which both allows for earlier diagnosis of BPD in infants dying before $28 d$ [36], and eliminates the problem of different interpretations of the terminology "at least $28 \mathrm{~d}$ " [27].

BPD severity is currently assessed at 36 weeks' PMA [8, 24], but when to best assess BPD severity remains unclear. Isayama showed that the assessment of BPD severity at 40 weeks' PMA had a higher predictive value for long-term negative pulmonary and neurosensory outcomes at $18-21$ months' corrected age [37]. However, neither 36 weeks' or 40 weeks' PMA are ideal time points, as a high percentage of infants are already discharged or transferred from tertiary level neonatal intensive care units by that age. Consequently, infants can be over- or under-classified as follow-up data are often missing after transfer to a regional unit $[36,38]$. The most recent NICHD classification retains the 36 w PMA time-point, acknowledging the movement of infants from the diagnostic centre beyond this time.

\section{7) Lack of distinction between underlying pathophysiologies of BPD}

Infants requiring invasive ventilation, N-CPAP, NIPPV or nasal cannula with flows above $3 \mathrm{~L} / \mathrm{min}$ are categorized as having BPD independent a requirement for supplemental oxygen by the most recent suggested BPD definition [24]. The dependency on respiratory support without the need for supplemental oxygen is most likely an indicator of small and/or large airway pathology, or of 
disrupted control of breathing and secondary obstructive airways consequent to primary central apnoea. Similarly, it is possible that some infants receiving minimal pressure nasal cannula support may only have an oxygen deficiency arising from reversible atelectasis that is consequent to airway collapse. The clinical management and future implications of each of these conditions is different, and is confused by a non-specific collective diagnosis of BPD that mixes pulmonary and nonpulmonary pathology into the definition of BPD. This pitfall persists in the most recent revision of the classification despite the ill-defined requirement for radiographic confirmation of parenchymal lung disease (e.g., when?; for how long?).

\section{8) Poor predictive value of current definitions for future respiratory morbidity and responsiveness to therapy.}

The predictive value for long-term pulmonary outcomes of the most recent proposed revision of the BPD definition is unknown but most likely will be plagued by the same issues facing earlier definitions. The 2001 BPD definition and the physiological classification proposed by Walsh have poor predictive value for long-term pulmonary outcomes [38, 39], as infants with and without BPD experience negative pulmonary long-term negative sequelae [40-45]. A composite outcome of respiratory support and oxygen therapy at 40 weeks' PMA had the highest predictive value for longterm pulmonary and neurosensory outcomes [37]. However, as respiratory support might reflect abnormal central nervous system function, the higher predictive value for negative neurosensory outcomes is not surprising. Alternatively, it may be a consequence of repeated hypoxia due to obstructive apnoea associated with airway disorders, unrelated to parenchymal pathology. Retrospective evaluations of the predictive value of objective neonatal variables for adverse later respiratory outcomes may inform new definitions. Such variables would require validation across multiple cohort sets, and may vary for different adverse respiratory outcomes. Predictive variables informed by later adverse outcomes would enhance planning for appropriate clinical follow-up and early intervention after hospital discharge. 


\section{The way forward: A dynamic definition of BPD based on pathophysiology?}

\section{Desired features of a new definition of BPD}

A new definition of BPD should strive to meet multiple goals: the definition should incorporate simple objective functional assessments ideally performed bedside by clinical staff to facilitate global uptake; further, the definition should be simple to interpret and apply, reflect the underlying pathophysiology of the disease and be adaptable to different stages of an evolutionary disease process. More than one bedside assessment tool may be necessary to achieve these objectives. The assessment tools require sufficient sensitivity to detect small improvements or deteriorations in disease severity at the population level (for evaluation of efficacy of new therapies); and additionally be sufficiently repeatable and reproducible to identify deterioration or improvement at the individual level. Finally, the assessment tool and ultimately the severity classifications within the definition of BPD, should have a high predictive value for negative pulmonary long-term sequelae to identify infants at high risk, guide parent discussions and prescribe treatment and follow-up options $[21,23,36]$. Meeting the objective of a dynamic definition, adaptable to different stages of the disease, requires flexibility in time-points of assessment according to the goal of classification at each stage. Identification of simple tests that can be applied in this dynamic fashion is key to achievement of these priorities. A tool that fulfils these criteria could be used for benchmarking neonatal units across the world and for the assessment of BPD in epidemiologic studies [42].

\section{A proposed new measure of the severity of BPD at the parenchymal level}

Shift of the oxyhaemoglobin dissociation curve addresses many of the desired features of a new definitive test for the presence and severity of BPD. Shift was first measured by Smith and Jones in neonates with pulmonary failure [46]. Shift is a continuous measure of pulmonary gas exchange capacity and can be assessed non-invasively at any PMA [47-49]. Similar to the oxygen reduction test proposed by Walsh, the $\mathrm{P}_{1} \mathrm{O}_{2}$ is reduced stepwise (at least 4 measurements) to achieve a $\mathrm{SpO}_{2}$ in the range of $96-88 \%$ (Figure $2 \mathrm{~A}$ ). A curve is fitted to the paired $\mathrm{SpO}_{2} / \mathrm{P}_{1} \mathrm{O}_{2}$ measurements and compared to the ideal oxyhaemoglobin dissociation curve. Shift reflects how much the $\mathrm{SpO}_{2} / \mathrm{P}_{1} \mathrm{O}_{2}$ curve is shifted to the right compared to the oxyhaemoglobin dissociation curve (Figure 2B). 
$2 A$

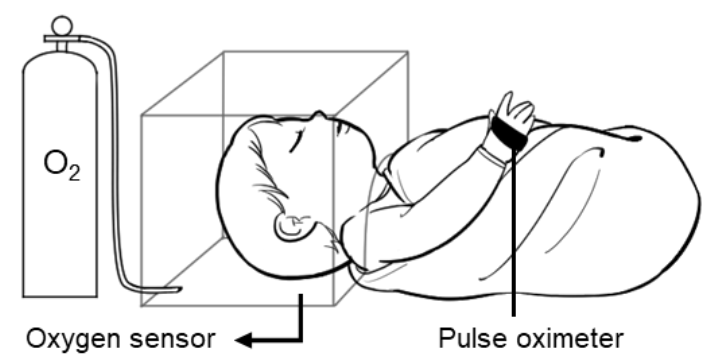

2B

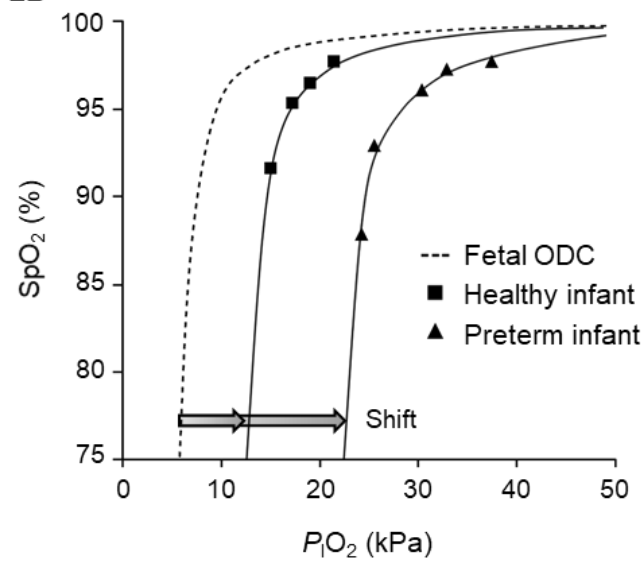

Figure 2. Assessment of the right shift of the $\mathrm{SpO}_{2} v s \mathrm{P}_{1} \mathrm{O}_{2}$ curve: A) the test is performed using a headbox, within which the $\mathrm{P}_{1} \mathrm{O}_{2}$ is adjusted stepwise. $\mathrm{SpO}_{2}$ is measured on the right hand at least 4 minutes after $\mathrm{P}_{1} \mathrm{O}_{2}$ has stabilised at each level. B) The paired measurements of $\mathrm{SpO}_{2}$ at each $\mathrm{P}_{1} \mathrm{O}_{2}$ are plotted, and the rightward shift of the $\mathrm{SpO} \mathrm{O}_{2} / \mathrm{P}_{1} \mathrm{O}_{2}$ curve from the oxyhaemoglobin dissociation curve is determined (arrows). The curve of a healthy term infant is shifted to the right by $\sim 6 \mathrm{kPa}$, whereas the rightward shift in the preterm infant depends on the infant's pulmonary gas exchange capacity. $\mathrm{SpO}_{2}$, peripheral oxyhaemoglobin saturation; $\mathrm{P}_{1} \mathrm{O}_{2}$, partial pressure of inspired oxygen.

This complete measurement of shift requires an extended period of assessment ( $45 \mathrm{~min}$ ), and may be suitable for definitive measurement on an individual infant. However, such extended measurements are resource intensive and hence impractical for global utilisation as a measurement of impaired parenchymal gas exchange.

Fortunately, the rightward shift of the oxyhaemoglobin dissociation curve can be predicted from a single paired $\mathrm{SpO}_{2} / \mathrm{P}_{1} \mathrm{O}_{2}$ measurement [50]. The use of a single paired $\mathrm{SpO}_{2} / \mathrm{P}_{1} \mathrm{O}_{2}$ measurement with a $\mathrm{SpO}_{2}$ around $90 \%$ allows a rapid and reliable assessment in most preterm infants at a common PMA. Both $\mathrm{SpO}_{2}$ and $\mathrm{P}_{1} \mathrm{O}_{2}$ are measured over a pre-defined period of time and the averaged $\mathrm{SpO}_{2} / \mathrm{P}_{1} \mathrm{O}_{2}$ measurement is used to determine shift from a lookup table [50]. The current look-up table for shift from a single paired $\mathrm{SpO}_{2} / \mathrm{P}_{1} \mathrm{O}_{2}$ measurement published by Quine et al. in 2006 is based on the adult oxyhaemoglobin dissociation curve and only allows determination of shift in infants with a $\mathrm{SpO}_{2} \leq 94 \%$ at a $P_{1} \mathrm{O}_{2} 21 \mathrm{kPa}$ [50]. A look-up table for infants with $\mathrm{SpO}_{2}>94 \%$, independent of the $P_{1} \mathrm{O}_{2}$ is needed to avoid hypoxic testing for the determination of shift in infants breathing room air.

The use of shift for objective assessment of the pulmonary parenchymal disruption in BPD has at least five key advantages. 1) The single paired measurement of $\mathrm{SpO}_{2}$ and $\mathrm{P}_{1} \mathrm{O}_{2}$ is a simple assessment 
with global applicability requiring minimal resources. 2) Shift is independent of differences in oxygen prescribing policies across neonatal units and is independent of altitude, as $\mathrm{P}_{1} \mathrm{O}_{2}$ reflects the $\mathrm{F}_{1} \mathrm{O}_{2}$ adjusted for altitude above sea level, as exemplified in Table 3. 3) Shift can be measured whilst preterm infants are on invasive and non-invasive respiratory support, hence has potential to be used across the evolution of BPD as a prospective determinant of BPD, as well as at more traditional assessment time-points. The theoretical impact of ventilator pressures on the calculation of shift are negligible compared to the impact of atmospheric pressure at different altitudes. When respiratory support is maintaining airway patency, the shift measurement will determine the gas exchange capacity of the parenchyma, rather than the consequences of airway collapse. 4) As a continuous outcome measure, shift potentially offers improved sensitivity for detection of change in disease severity at both individual and population levels. 5) Finally, shift of the oxyhaemoglobin dissociation curve offers a physiological assessment of BPD disease severity at the parenchymal level and would therefore add valuable information to the current BPD definitions.

Despite the apparent advantages of using shift of the $\mathrm{SpO}_{2}$ versus $\mathrm{P}_{1} \mathrm{O}_{2}$ curve for equitable determination of parenchymal diseases severity, more research is required before shift can be incorporated meaningfully into a future definition of BPD. Research studies need to explore the impact of invasive and non-invasive ventilator pressures and flows on the derived measures of shift and capacity for pulmonary gas exchange $[51,52]$. Reference values for different respiratory support modes may be required. Short-term repeatability and reliability of shift need to be assessed to identify its utility for tracking disease severity at the individual level, and the long-term prognostic value of shift requires long-term follow-up studies. Establishment of reference ranges for shift across the full range of PMA and/or PNA could enable early identification of preterm infants with evolving severe BPD who might benefit from novel and potentially life-saving interventions. Future versions of the look-up table for the single paired measurement of $\mathrm{SpO}_{2}$ and $\mathrm{P}_{1} \mathrm{O}_{2}$ will likely adjust shift values for fetal haemoglobin to facilitate more accurate estimation of shift prior to term, and expand the table to include estimations of shift for infants with $\mathrm{SpO}_{2}>90 \%$ when breathing air.

\section{Conclusion}

The current BPD definitions including the oxygen reduction test proposed by Walsh lead to misclassifications of preterm infants with and without BPD, and do not allow reliable prediction of adverse long-term pulmonary outcomes. Right shift of $\mathrm{SpO}_{2} / \mathrm{P}_{1} \mathrm{O}_{2}$ curve is a continuous objective variable of pulmonary gas exchange capacity that is independent of oxygen prescribing policies 
across neonatal units and altitude. Assessing pulmonary gas exchange capacity in preterm infants allows an improved understanding of the underlying pathophysiology of BPD. However, BPD is most likely a compound of cardiac complications, diseases of the conductive airways, immaturity of the respiratory muscles and immature control of breathing. Similar functional tests should aim to identify their respective contribution to BPD. Nevertheless, the assessment of shift of the $\mathrm{SpO}_{2} / \mathrm{P}_{1} \mathrm{O}_{2}$ curve could be used globally for the assessment of disease severity in preterm infants with BPD. The simplified assessment of shift from a single paired $\mathrm{SpO}_{2} / \mathrm{P}_{1} \mathrm{O}_{2}$ measurement could be used as a benchmarking tool for neonatal units and also as an outcome variable for clinical trials investigating BPD in preterm infants.

\section{Acknowledgements}

We would like to thank Jane Choi for the help with the design of figure 2.

\section{References}

1. Stoll, B.J., et al., Trends in Care Practices, Morbidity, and Mortality of Extremely Preterm Neonates, 1993-2012. JAMA, 2015. 314(10): p. 1039-1051.

2. Northway, W.H., Jr., R.C. Rosan, and D.Y. Porter, Pulmonary disease following respirator therapy of hyaline-membrane disease. Bronchopulmonary dysplasia. N Engl J Med, 1967. 276(7): p. 357-68.

3. Taghizadeh, A. and E.O. Reynolds, Pathogenesis of bronchopulmonary dysplasia following hyaline membrane disease. The American Journal of Pathology, 1976. 82(2): p. 241-264.

4. McEvoy, C.T. and J.L. Aschner, The Natural History of Bronchopulmonary Dysplasia (BPD): The Case for Primary Prevention. Clinics in perinatology, 2015. 42(4): p. 911-931.

5. Workshop on bronchopulmonary dysplasia. Sponsored by the Division of Lung Diseases. National Heart, Lung, and Blood Institute, National Institutes of Health. J Pediatr, 1979. 95(5 Pt 2): p. 1-9, 815-920.

6. Tooley, W.H., Epidemiology of bronchopulmonary dysplasia. J Pediatr, 1979. 95(5 Pt 2): p. 851-8.

7. Bancalari, E., et al., Bronchopulmonary dysplasia: clinical presentation. J Pediatr, 1979. 95(5 Pt 2): p. 819-23.

8. Jobe, A.H. and E. Bancalari, Bronchopulmonary dysplasia. Am J Respir Crit Care Med, 2001. 163(7): p. 1723-9. 
9. Shennan, A.T., et al., Abnormal Pulmonary Outcomes in Premature Infants: Prediction From Oxygen Requirement in the Neonatal Period. Pediatrics, 1988. 82(4): p. 527-532.

10. Burri, P.H., Structural Aspects of Postnatal Lung Development-Alveolar Formation and Growth. Neonatology, 2006. 89(4): p. 313-322.

11. Jobe, A.J., The New BPD: An Arrest of Lung Development. Pediatric Research, 1999. 46: $\mathrm{p}$. 641.

12. Joshi, S. and S. Kotecha, Lung growth and development. Early Human Development, 2007. 83(12): p. 789-794.

13. Husain, A.N., N.H. Siddiqui, and J.T. Stocker, Pathology of arrested acinar development in postsurfactant bronchopulmonary dysplasia. Human Pathology, 1998. 29(7): p. 710-717.

14. Hislop, A.A., et al., The effects of preterm delivery and mechanical ventilation on human lung growth. Early Hum Dev, 1987. 15(3): p. 147-64.

15. Albertine, K.H., et al., Chronic lung injury in preterm lambs. Disordered respiratory tract development. Am J Respir Crit Care Med, 1999. 159(3): p. 945-58.

16. Coalson, J.J., V. Winter, and R.A. deLemos, Decreased alveolarization in baboon survivors with bronchopulmonary dysplasia. Am J Respir Crit Care Med, 1995. 152(2): p. 640-6.

17. Berger, T.M., M. Fontana, and M. Stocker, The journey towards lung protective respiratory support in preterm neonates. Neonatology, 2013. 104(4): p. 265-74.

18. Walsh, M.C., et al., Safety, reliability, and validity of a physiologic definition of bronchopulmonary dysplasia. J Perinatol, 2003. 23(6): p. 451-6.

19. Mahle, W.T., et al., Endorsement of Health and Human Services Recommendation for Pulse Oximetry Screening for Critical Congenital Heart Disease. Pediatrics, 2012. 129(1): p. 190192.

20. Ibrahim, J. and V. Bhandari, The definition of bronchopulmonary dysplasia: an evolving dilemma. Pediatr Res, 2018.

21. Jensen, E.A. and C.J. Wright, Bronchopulmonary Dysplasia: The Ongoing Search for One Definition to Rule Them All. J Pediatr, 2018. 197: p. 8-10.

22. Babata, K. and J. McGuirl, Are we ready to modify our definition of bronchopulmonary dysplasia (BPD) to improve prognostication? J Perinatol, 2018. 38(3): p. 203-205.

23. Bancalari, E. and D. Jain, Bronchopulmonary Dysplasia: Can We Agree on a Definition? Am J Perinatol, 2018. 35(6): p. 537-540. 
24. Higgins, R.D., et al., Bronchopulmonary Dysplasia: Executive Summary of a Workshop. J Pediatr, 2018. 197: p. 300-308.

25. McEvoy, C.T., et al., Bronchopulmonary dysplasia: NHLBI Workshop on the Primary Prevention of Chronic Lung Diseases. Ann Am Thorac Soc, 2014. 11 Suppl 3: p. S146-53.

26. van Rossem, M.C., et al., Accuracy of the Diagnosis of Bronchopulmonary Dysplasia in a Referral-Based Health Care System. The Journal of Pediatrics, 2015. 167(3): p. 540-544.e1.

27. Ellsbury, D.L., et al., Variability in the use of supplemental oxygen for bronchopulmonary dysplasia. J Pediatr, 2002. 140(2): p. 247-9.

28. Sahni, R., et al., Is the new definition of bronchopulmonary dysplasia more useful? J Perinatol, 2005. 25(1): p. 41-6.

29. Strueby, L. and B. Thebaud, Advances in bronchopulmonary dysplasia. Expert Rev Respir Med, 2014. 8(3): p. 327-38.

30. Baillie, J.K. www.altitude.org. 2010 [cited 2017 30/11/2017].

31. Peacock, A.J., Oxygen at high altitude. BMJ : British Medical Journal, 1998. 317(7165): p. 1063-1066.

32. Curran-Everett, D., A classic learning opportunity from Fenn, Rahn, and Otis (1946): the alveolar gas equation. Advances in Physiology Education, 2006. 30(2): p. 58-62.

33. Britton, J.R., Altitude, oxygen and the definition of bronchopulmonary dysplasia. J Perinatol, 2012. 32(11): p. 880-5.

34. Gulliver, K. and B.A. Yoder, Bronchopulmonary dysplasia: effect of altitude correction and role for the Neonatal Research Network Prediction Algorithm. J Perinatol, 2018. 38(8): p. 1046-1050.

35. Jobe, A.H., The new BPD. NeoReviews, 2006. 7(10): p. e531-e545.

36. Jobe, A.H. and E.H. Bancalari, Controversies about the definition of bronchopulmonary dysplasia at 50 years. Acta Paediatr, 2017. 106(5): p. 692-693.

37. Isayama, T., et al., Revisiting the Definition of Bronchopulmonary Dysplasia: Effect of Changing Panoply of Respiratory Support for Preterm Neonates. JAMA Pediatr, 2017. 171(3): p. 271-279.

38. Poindexter, B.B., et al., Comparisons and Limitations of Current Definitions of Bronchopulmonary Dysplasia for the Prematurity and Respiratory Outcomes Program. Ann Am Thorac Soc, 2015. 12(12): p. 1822-30. 
39. Onland, W., et al., Clinical prediction models for bronchopulmonary dysplasia: a systematic review and external validation study. BMC Pediatr, 2013. 13: p. 207.

40. Simpson, S.J., et al., Altered lung structure and function in mid-childhood survivors of very preterm birth. Thorax, 2017. 72(8): p. 702-711.

41. Fawke, J., et al., Lung function and respiratory symptoms at 11 years in children born extremely preterm: the EPICure study. Am J Respir Crit Care Med, 2010. 182(2): p. 237-45.

42. Ehrenkranz, R.A., et al., Validation of the National Institutes of Health consensus definition of bronchopulmonary dysplasia. Pediatrics, 2005. 116(6): p. 1353-60.

43. Urs, R., et al., Persistent and progressive long-term lung disease in survivors of preterm birth. Paediatr Respir Rev, 2018.

44. J., S.S., H.G. L., and W.A. C., Lung function following very preterm birth in the era of 'new' bronchopulmonary dysplasia. Respirology, 2015. 20(4): p. 535-540.

45. Simpson, S.J., et al., Lung function trajectories throughout childhood in survivors of very preterm birth: a longitudinal cohort study. The Lancet Child \& Adolescent Health, 2018. 2(5): p. 350-359.

46. Smith, H.L. and J.G. Jones, Non-invasive assessment of shunt and ventilation/perfusion ratio in neonates with pulmonary failure. Arch Dis Child Fetal Neonatal Ed, 2001. 85(2): p. F12732.

47. Sapsford, D.J. and J.G. Jones, The PIO2 vs. SpO2 diagram: a non-invasive measure of pulmonary oxygen exchange. Eur J Anaesthesiol, 1995. 12(4): p. 375-86.

48. Lockwood, G.G., N.L. Fung, and J.G. Jones, Evaluation of a computer program for noninvasive determination of pulmonary shunt and ventilation-perfusion mismatch. J Clin Monit Comput, 2014. 28(6): p. 581-90.

49. Jones, J.G. and S.E. Jones, Discriminating between the effect of shunt and reduced VA/Q on arterial oxygen saturation is particularly useful in clinical practice. J Clin Monit Comput, 2000. 16(5-6): p. 337-50.

50. Quine, D., et al., Non-invasive measurement of reduced ventilation:perfusion ratio and shunt in infants with bronchopulmonary dysplasia: a physiological definition of the disease. Arch Dis Child Fetal Neonatal Ed, 2006. 91(6): p. F409-14.

51. Iyer, N.P. and M.J. Mhanna, Association Between High-Flow Nasal Cannula and EndExpiratory Esophageal Pressures in Premature Infants. Respiratory Care, 2016. 61(3): p. 285290. 
52. Lampland, A.L., et al., Observational Study of Humidified High-Flow Nasal Cannula Compared with Nasal Continuous Positive Airway Pressure. The Journal of Pediatrics, 2009. 154(2): p. 177-182.e2. 